\title{
Higher order Hermite enriched contact finite elements for adhesive contact problems
}

\author{
Vishal Agrawal and Sachin Singh Gautam* \\ Department of Mechanical Engineering, \\ Indian Institute of Technology Guwahati, \\ Guwahati, India \\ Fax: +361-258-2699 \\ Email: v.agrawal@iitg.ernet.in \\ Email:ssg@iitg.ernet.in \\ *Corresponding author
}

\begin{abstract}
It is known that during the simulation of the adhesive contact problems highly nonlinear responses of interaction forces occur within the very narrow adhesive zone. It leads to the loss of quadratic-rate of convergence during Newton-Raphson iterations and unstable computational behaviour. In case of standard finite element formulation, a very fine mesh resolution is needed for the stable computations, but a significant computational cost is associated. For minimising the cost without the loss of accuracy of the solution, contact surface enrichment approaches have been presented. These approaches utilise the higher-order Lagrangian polynomial functions for the enrichment of contact finite elements. In the present work, based on the incorporation of fifth- and seventh-order Hermite interpolation functions two new enriched contact finite elements are formulated. The performance of proposed enriched contact finite elements is demonstrated through the simulation of peeling of an initially flat deformable strip from a rigid substrate. A stable solution is obtained at a relatively coarser mesh than the fully Lagrangian discretised finite element mesh. It is shown that the proposed higher order Hermite enriched contact finite elements attain better performance when compared with earlier introduced enriched elements.
\end{abstract}

Keywords: adhesion; computation contact mechanics; enriched finite elements; nonlinear finite element analysis; Hermite polynomials.

Reference to this paper should be made as follows: Agrawal, V. and Gautam, S.S. (2019) 'Higher order Hermite enriched contact finite elements for adhesive contact problems', Int. J. Materials and Structural Integrity, Vol. 13, Nos. 1/2/3, pp.16-31.

Biographical notes: Vishal Agrawal is presently a $\mathrm{PhD}$ student in the Department of Mechanical Engineering, Indian Institute of Technology Guwahati, India. His area of research is the study of large deformation contact problems using the NURBS based isogeometric analysis.

Sachin Singh Gautam is currently an Assistant Professor in the Department of Mechanical Engineering, Indian Institute of Technology Guwahati, India. He received his MTech and $\mathrm{PhD}$ degrees in Mechanical Engineering discipline from Indian Institute of Technology Kanpur, India. He has also been a Postdoctoral Fellow and visiting researcher at RWTH Aachen University, Aachen, Germany. His current research interest includes nonlinear finite element analysis, computational contact mechanics, and isogeometric analysis. $\mathrm{He}$ is a member of ASME and DYMAT. 
This paper is a revised and expanded version of a paper entitled 'Enrichment of finite elements with higher order hermite polynomials for adhesive contact problems' presented at Indian Conference on Applied Mechanics (INCAM) Conference, MNIT Allahabad, India, 5-7 July 2017.

\section{Introduction}

Contact problems are considered to be the most complex as numerical methods are often the only available approach for their study (Laursen, 2002; Wriggers, 2006). Adhesive contact problems are characterised by the nano-scale contact problems in which the interacting bodies are strongly affected by the distance-dependent inter-molecular forces (Israelachvili, 1991). Understanding the adhesion mechanism of the peeling of gecko spatula (a toe of gecko has millions of nano-sized spatula-shaped hairs which interact with underlying surface and enable them to cling on any surface, see Sauer and $\mathrm{Li}$ (2007a) for further details) has been a fascinating topic for researchers for a very long time. Autumn et al. (2002) found that the adhesion mechanism of gecko setae (each setae has hundreds of spatula-shaped projections, see Figure 12 from Gautam and Sauer, 2013) can be appropriately described by the van der Waals adhesion model. Since then, the research in this field is accelerating rapidly, and van der Waals model is rigorously used for the study adhesive problems. For predicting the behaviour of deformable bodies under the influence of intermolecular van der Waals forces, Sauer and Li (2007a, 2007b) and Sauer and Wriggers (2009) presented a generalised finite element based computational contact formulation for the analysis of various adhesive contact problems. Sauer (2008) and Sauer and Holl (2013) presented the numerical simulation of the peeling process of a gecko spatula. Later, Gautam and Sauer $(2014 ; 2013)$ presented the composite and energy-momentum conserving time-integration algorithms for the dynamic analysis of gecko-spatula peeling problems. However, one of the major issues with van der Waals model is that the intermolecular forces are of highly nonlinear nature and show huge jumps in their values even for a small change in the distance of interacting molecules (Israelachvili, 1991). It is observed that during the numerical simulation of adhesive contact problems using the standard bilinear Lagrangian interpolation based finite element (FE) discretisation highly oscillatory responses of adhesive forces, which are considered as numerical artefacts, are present and strongly affects the rate of convergence of Newton-Raphson iterations. As a result, it leads to incorrect computational behaviour (Sauer, 2011). An inappropriate discretisation of the body leads to the failure of simulation (see Figure 13(a) from Gautam and Sauer, 2013). For the stable computation and accurate solution of adhesive problems, a very fine FE mesh resolution is needed to minimise or alleviate the issues due to the large jumps in the value of van der Waals adhesive forces. However, the use of highly refined mesh increases the computational cost substantially, which may not be the likely case in the FE community. In order to improve the approximation of adhesive forces at a lower computation cost or coarser, a mesh level surface enrichment approach has been presented (Sauer, 2011). In that, higher-order Lagrangian and cubic-order Hermite polynomial functions have been used to locally introduce the additional unknowns or degrees-of-freedom (DOF) over the interaction surface of contact finite elements. Another approach to alleviate the boundary 
effects is presented in Ge et al. (2016). Later, NURBS and T-spline basis functions, which represent the exact form of interaction surface, have also been used to accurately capture the high gradient adhesive forces (Corbett and Sauer, 2014, 2015).

In the present work, two new enriched contact finite elements, based on the incorporation of fifth- and seventh-order Hermite interpolation functions, are formulated to attenuate the issues stemming from the highly nonlinearity and large gradient of adhesive forces. The idea is based on the fact that the Hermite polynomial based FE formulation provides the smooth representation of surfaces due to their $\mathrm{C}^{1}$ - continuity and offer more number of DOF at each node in comparison to Lagrangian based FE formulation. The structure of this paper is organised as follows: Section 2 presents a brief overview of the formulation for adhesive contact problem in the framework of computational contact mechanics. Section 3 provides the formulation for the newly developed enriched contact finite elements. A numerical example is discussed in Section 4. The results along with the relevant discussion are described in Section 5. Finally, Section 6 presents the conclusions with future directions.

Figure 1 Contact kinematics of a deformable body $\mathcal{B}$ and rigid surface combination
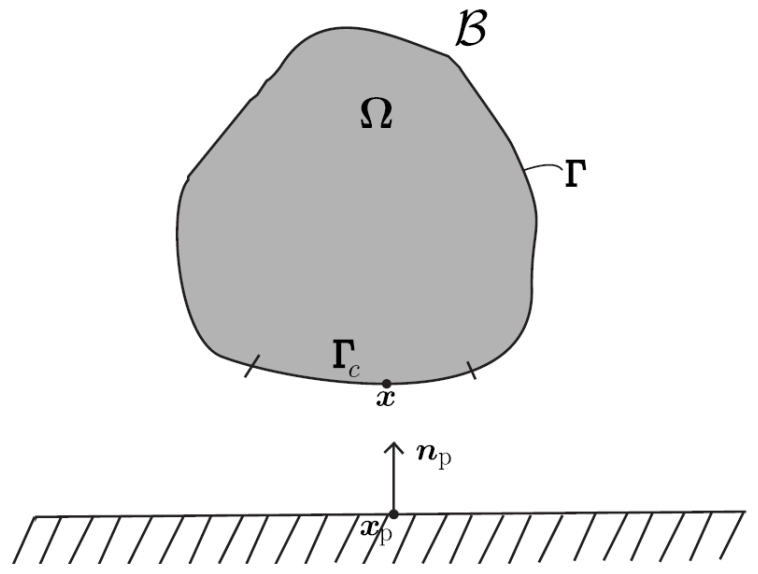

\section{Formulation}

This section is divided into two subsections. In the first, a brief overview of continuum based formulation of adhesion model is presented. After that, weak formulation of adhesive contact problem and its finite element based discretisation are presented in the second subsection.

\subsection{Adhesion formulation}

The fundamental concept that is used to describe the adhesion mechanism between the deformable contacting bodies is to determine the interaction potential function $\Phi$, which accounts for the local interaction of each particle of contacting bodies. For the determination of $\Phi$, the body force (BF) and surface force (SF) formulation approaches are introduced in the literature (Sauer and Li, 2007a; Sauer and Lorenzis, 2013). In the 
present work, we take advantage of SF approach for the numerical modelling of adhesive contact problem. For describing the interaction mechanism, van der Waals adhesion model is used, and the formulation is done based on the updated Lagrangian description.

Consider a body $\mathcal{B}$ in its current configuration having domain $\Omega$, surface $\Gamma$, and the part of its boundary where contact takes place as $\Gamma_{c}$. The arrangement is shown in Figure 1. The interaction between the deformable body $\mathcal{B}$ and the rigid body pair can be described as (Sauer and Li, 2007a):

$$
\Pi_{c}=\int_{\Gamma_{c}} \beta \Phi d \Gamma
$$

where $\beta$ is the surface density of interacting particles over the per unit current surface area of body $\mathcal{B}$ at $x \in \Gamma_{c}$, and interaction potential $\Phi$ depends on the minimum distance $r_{m}(x)$ between the point $\mathrm{x}$ and rigid surface as:

$$
\begin{aligned}
& r_{m}(x):=\min \left\|x-x_{p}\right\| \\
& r_{m}(x)=\left(x-x_{p}\right) \cdot n_{p}
\end{aligned}
$$

where $x_{p}$ and $\boldsymbol{n}_{p}$ denote the position and normal unit vector at the closest projection point of $x \in \Gamma_{c}$ on the rigid surface. In this formulation it is considered that the number of interaction particles remains conserved over the surface during the deformation (Sauer and Wriggers, 2009). This assumption is hold true for solid bodies. The variation of interaction energy, due to the deformation in the configuration of $\mathcal{B}$, represented by $\delta \boldsymbol{x}=\delta \varphi$ is given as

$$
\delta \Pi_{c}=\int_{\Gamma_{c}} \boldsymbol{T}_{c} \cdot \delta \boldsymbol{\varphi} d \Gamma
$$

In this equation, $\boldsymbol{T}_{c}$ denotes the surface traction and is defined as

$$
\boldsymbol{T}_{c}(x)=\beta \boldsymbol{F}_{I}(x) \quad \text { where } \boldsymbol{F}_{I}(x)=-\frac{\partial \Phi}{\partial x}
$$

Here, $\boldsymbol{F}_{I}(x)$ is the interaction force. For the van der Waals adhesion model, the surface traction is obtained by integrating the Lennard-Jones potential four times and is given as (Israelachvili, 1991; Sauer and Li, 2007a):

$$
\boldsymbol{T}_{c}(x)=\frac{A_{H}}{2 \pi r_{0}^{3}}\left[\frac{1}{45}\left(\frac{r_{0}}{r_{m}}\right)^{9}-\frac{1}{3}\left(\frac{r_{0}}{r_{m}}\right)^{3}\right] \cdot n_{p}
$$

where the parameters $r_{0}$ and $A_{H}$ are identified as the equilibrium distance of interacting particles, and as the Hamaker constant for Lennard-Jones potential, respectively. $\boldsymbol{T}_{c}(x)$ denotes the adhesive traction vector, which accounts for the attraction or repulsion forces over the contact elements. It is a function of normal unit vector $n_{p}$ and the minimum gap $r_{m}(x)$ between the interaction points of contacting bodies. The major difficulty with its expression is that a very fine mesh resolution is needed to accurately capture the large gradients in the value of interaction forces. When the minimum gap is equal to the equilibrium distance of the adhesion model $\left(r_{m}(x)=r_{e q}\right)$, the traction vector goes to infinity (Israelachvili, 1991). Also, when the minimum gap is a below to the equilibrium 
distance $\left(r_{m}(x)<r_{e q}\right)$, the traction forces vary extremely rapidly due to the very high slope value of contact traction for the minimum gap (within the repulsive zone of the adhesion model). For fixing these issues, the adhesive traction is regularised, where the slope is fixed to a constant value when the minimum gap is found below to equilibrium distance of the model (Sauer, 2011). For the regularisation, penalty method is used in the following manner:

$$
\boldsymbol{T}_{c}(x)=\left\{\begin{array}{cl}
-\varepsilon_{n} r_{m} n_{p} & r_{m}<r_{e q} \\
0 & r_{m} \geq r_{e q}
\end{array}\right.
$$

In the above equation $\varepsilon_{n}$ denotes the penalty parameter and $r_{m}$ is treated as the normal gap between the interacting bodies.

\subsection{Weak formulation and finite element discretisation}

In this section, we outline the weak formulation for the frictionless, quasi-static adhesive contact problem and its displacement based discretisation in the context of finite element method.

The governing equation for the considered problem is given by the equilibrium between the work done by the internal, external, and contact forces. From the admissible variation of deformation function $\delta \varphi$, the weak form of adhesive contact for a deformable body is given by the following statement:

$$
\int_{\Omega} \operatorname{grad}(\delta \boldsymbol{\varphi}): \sigma d \Omega-\int_{\Gamma_{c}} \delta \boldsymbol{\varphi} \cdot \boldsymbol{T}_{c} d \Gamma-\delta \Pi_{\mathrm{ext}}=0 \quad \forall \delta \boldsymbol{\varphi} \in \mathcal{V}
$$

where $\mathcal{V}$ is the space of kinematically admissible function $\delta \varphi$ and $\sigma$ is the Cauchy stress tensor, which follows from the constitutive equations. In this work, a Neo-Hookean hyperelastic material model is considered, and the stress tensor is determined by the following expression (Bonet and Wood, 1997):

$$
\boldsymbol{\sigma}=\frac{\lambda}{J} \ln J \boldsymbol{I}+\frac{\mu}{J}\left(\boldsymbol{F} \boldsymbol{F}^{T}-\boldsymbol{I}\right)
$$

where $\lambda$ and $\mu$ are Lamé's constant, $\boldsymbol{I}$ is the second order identity tensor and $J$ denotes the determinant of deformation gradient tensor $\boldsymbol{F}$. Following a displacement based FE formulation (Hughes, 2000; Wriggers, 2006), the domain of the contact body is discretised into a number of elements such that $\Omega=\sum_{e=1}^{n} \Omega^{e}$, where $n$ represent the total number of finite elements. The displacement field $u^{e}$ and the variation $\delta \varphi$ is given by the interpolation of FE basis function as:

$$
u^{e}=\sum_{i=1}^{n^{e}} N_{i} u_{i}=\mathbf{N u} \text { and } \varphi^{e}=\mathbf{N v}
$$

In the above equations, $\mathbf{u}$ and $\mathbf{v}$ represents the nodal displacement and virtual displacement vector of FE nodes, and $\mathbf{N}$ is the matrix of FE shape functions as $\mathbf{N}=\left[N_{1} \mathbf{I}, N_{2} \mathbf{I}, \ldots, N_{n^{e}} \mathbf{I}\right]$. Here, $n^{e}$ denotes the number of nodes per element and $\mathbf{I}$ is an 
identity matrix. The reference and the current configuration of interacting body are described likewise to the displacement field shown as in equations (10). Based on this discretisation, the weak form for adhesive contact, equation (8), can be cast into the following finite element form

$$
\mathbf{v}^{T}\left[\mathbf{f}_{\text {int }}(u)+\mathbf{f}_{c}(u)-\mathbf{f}_{\text {ext }}\right]=0
$$

where $\mathbf{f}_{\text {int }}, \mathbf{f}_{c}$ and $\mathbf{f}_{\text {ext }}$ are the vectors for internal, contact, and externally applied forces, respectively. The first term represents the virtual work done by the internal force vector, which is used to describe the bulk behaviour of given body. The computation procedure of the system stiffness matrix and force vector quantities that account the bulk behaviour can be followed from standard FE textbook (e.g., Bonet and Wood, 1997). The second term denotes the virtual work done by the contact forces. The contact force vector $\mathbf{f}_{c}$, which makes the contributions to the contact elements $\Omega^{e}$ of body $\mathcal{B}$, is computed by the following expression:

$$
\mathbf{f}_{c}(u)=\sum_{e=1}^{n} \mathbf{f}_{c}^{e}\left(u^{e}\right) \quad \text { where } \quad \mathbf{f}_{c}^{e}\left(u^{e}\right)=-\int_{\Gamma_{c}^{e}} \mathbf{N}^{T} \boldsymbol{T}_{c}\left(u^{e}\right) d \Gamma
$$

where $\mathbf{N}$ is the shape function matrix and formed using the basis functions for a four-node Lagrangian FE element for standard FE formulation. While in case of the enrichment with higher-order Hermite interpolation functions, it consists of the basis functions for the newly formulated enriched contact finite elements. To ease the evaluation of contact contribution, the integral in above equation is mapped to a master element $\Gamma_{c \square}^{e}$ using the standard FE procedure. The integral is then calculated with the numerical quadrature rule as:

$$
\mathbf{f}_{c}^{e}\left(u^{e}\right)=-\sum_{g p=1}^{n_{g p}} \mathbf{N}^{T}\left(\xi_{g p}\right) \boldsymbol{T}_{c}\left(\xi_{g p}\right) j\left(\xi_{g p}\right) w_{g p} \quad \xi_{g p} \in \Gamma_{c \square}
$$

where $n_{g p}$ denotes the number of quadrature points on the master surface, $w_{g p}$ are their respective weights and $j$ is the determinant of Jacobian matrix that enables the transformation of surface area $d \Gamma$. In order to improve the approximation of surface tractions at the coarse mesh level, equation (6) and its integration in equation (12), the interaction surface $\Gamma_{c \square}^{e}$ of master element is described with the higher-order Hermite interpolation function. The procedure is presented in the next section. Further details, including the computation of contact stiffness matrix using the contact force vector $\mathbf{f}_{c}$ can be found in (Sauer, 2011). In the numerical problem considered in this paper no external force is applied $\left(\mathbf{f}_{\mathrm{ext}}=0\right)$.

\section{Proposed enrichment strategy}

In this section, we first present the formulation of newly proposed higher-order Hermite enriched contact finite elements. Then, a brief review of higher-order Lagrangian enriched contact finite elements, which are used for comparing the performance of the proposed enrichment, is presented. 


\subsection{Hermite enriched contact finite elements}

In the proposed enrichment strategy the overall domain of interacting body is divided into the two parts. The first part consists of the contact element that includes the interaction surface of the body, and the second part includes the remaining bulk of domain that is free of interaction. For the purpose of visualisation of this arrangement, a representative image of a deformable strip is shown in the Figure 5. In this approach, the interaction surface of contact finite elements is locally enriched with the higher-order Hermite interpolation functions to improve the capturing ability of highly nonlinear van der Waals adhesive traction over the $\Gamma_{c \square}^{e}$. For this, first, the current configuration of a standard finite element $\Omega^{e}$ is mapped to a master element $\Omega_{\square}^{e}$. Then, the interaction surface $\Gamma_{c \square}^{e}$ of the master element $\Omega_{\square}^{e}$ is described with either the fifth- or seventh-order Hermite interpolation functions. The schematic arrangement of a fifth-order Hermite enriched contact finite element is shown in Figure 2. It consists of overall eight nodes: five standard FE nodes (node numbered 1 to 5) for the approximation of displacement field, and three additional nodes (node numbered 6 to 8 ) across the element's surface for displacement derivatives. The newly formulated fifth-order Hermite enriched element is represented by the Q1CH5, where the first, and last entry in the notation denotes the order of interpolation functions that are used to describe the bulk, and the contact surface, respectively. The displacement field for fifth-order Hermite smoothed element is given by the following expression:

$$
u^{e}=\sum_{i=1}^{5} N_{i} u_{i}+\sum_{i=1}^{3} H_{i} u_{i, \xi}
$$

The basis functions are derived based on the procedure presented in (Augardea, 1998) and are defined as:

$$
\begin{aligned}
& N_{1}(\xi, \eta)=\frac{1}{8} \xi^{2}(3 \xi+4)(1-\xi)^{2}(1-\eta) \\
& N_{2}(\xi, \eta)=\frac{1}{8} \xi^{2}(4-3 \xi)(1-\xi)^{2}(1-\eta) \\
& N_{5}(\xi, \eta)=\frac{1}{2}\left(1-\xi^{2}\right)^{2}(1-\eta) \\
& H_{1}(\xi, \eta)=\frac{1}{8} \xi^{2}(1+\xi)(1-\xi)^{2}(1-\eta) \\
& H_{2}(\xi, \eta)=\frac{1}{2} \xi\left(1-\xi^{2}\right)^{2}(1-\eta) \\
& H_{3}(\xi, \eta)=\frac{1}{8} \xi^{2}(\xi-1)(1+\xi)^{2}(1-\eta)
\end{aligned}
$$

According to the isoparametric concept, these functions are also used for the description of the reference and the current configuration of Q1CH5 element as

$$
\boldsymbol{X}^{e}=\sum_{i=1}^{5} N_{i} \boldsymbol{X}_{i}+\sum_{i=1}^{3} H_{i} \boldsymbol{X}_{i, \xi} \quad \text { and } \quad \boldsymbol{x}^{e}=\sum_{i=1}^{5} N_{i} \boldsymbol{x}_{i}+\sum_{i=1}^{3} H_{i} \boldsymbol{x}_{i, \xi}
$$


where $\boldsymbol{X}_{i}$ and $\boldsymbol{x}_{i}$ represents the nodal coordinate vectors of reference and current configuration for node $i$. In case of seventh-order Hermite enriched contact finite element, the interaction surface $\Gamma_{c \square}^{e}$ is described by the seventh-order Hermite interpolation function. The resulting element is denoted by Q1CH7 and is shown in Figure 3. The Q1CH7 element consists of six standard FE nodes for the displacement field (node numbered 1 to 6) and four nodes (node numbered 7 to 10) for the displacement derivatives across the surface $\Gamma_{c \square}^{e}$ of master element $\Omega_{\square}^{e}$. The displacement field for Q1CH7 element is given as:

$$
u^{e}=\sum_{i=1}^{6} N_{i} u_{i}+\sum_{i=1}^{4} H_{i} u_{i, \xi}
$$

The basis functions are defined as:

$$
\begin{aligned}
& N_{1}(\xi, \eta)=\frac{4}{54}(19 \xi+22)(1-\xi)^{2}\left(\frac{1}{4}-\xi^{2}\right)^{2}(1-\eta) \\
& N_{2}(\xi, \eta)=\frac{4}{54}(22-19 \xi)(1+\xi)^{2}\left(\frac{1}{4}-\xi^{2}\right)^{2}(1-\eta) \\
& N_{5}(\xi, \eta)=\frac{16}{27}(1-\xi)\left(\frac{1}{2}-\xi\right)^{2}\left(1-\xi^{2}\right)^{2}(1-\eta) \\
& N_{6}(\xi, \eta)=\frac{16}{27}(1+\xi)\left(\frac{1}{2}+\xi\right)^{2}\left(1-\xi^{2}\right)^{2}(1-\eta) \\
& H_{1}(\xi, \eta)=\frac{4}{18}(1-\xi)^{2}\left(\frac{1}{4}-\xi^{2}\right)^{2}(1+\xi)(1-\eta) \\
& H_{2}(\xi, \eta)=\frac{8}{9}\left(\xi+\frac{1}{2}\right)\left(\frac{1}{2}-\xi\right)^{2}\left(1-\xi^{2}\right)^{2}(1-\eta) \\
& H_{3}(\xi, \eta)=\frac{8}{9} \xi^{2}\left(\xi-\frac{1}{2}\right)\left(\frac{1}{2}+\xi\right)^{2}\left(1-\xi^{2}\right)^{2}(1-\eta) \\
& H_{4}(\xi, \eta)=\frac{4}{18}(\xi+1)^{2}\left(\frac{1}{4}-\xi^{2}\right)^{2}(\xi-1)(1-\eta)
\end{aligned}
$$

where the basis functions $N_{3}(, \xi \eta)$ and $N_{4}(, \xi \eta)$ are omitted from the equations (15) and (18), since they are same as for a standard bilinear FE element. The basis functions shown in equations (15) and (18) satisfy the partition of unity:

$$
\sum_{i} N_{i}(\xi, \eta)+\sum_{i} H_{i}(\xi, \eta)=1 \quad \forall \xi, \eta \in \Omega_{\square}^{e}
$$

and the compact support property:

$$
N_{i}\left(\xi_{j}, \eta_{j}\right)=\delta_{i j} \text { and } H_{i}\left(\xi_{j}, \eta_{j}\right)=0 \quad \forall \xi, \eta \in \Gamma_{c \square}^{e}
$$

at each node of contact elements. The geometry of Q1CH7 element within the reference and current configuration is described by the following expressions: 


$$
\boldsymbol{X}^{e}=\sum_{i=1}^{6} N_{i} \boldsymbol{X}_{i}+\sum_{i=1}^{4} H_{i} \boldsymbol{X}_{i, \xi} \text { and } \boldsymbol{x}^{e}=\sum_{i=1}^{6} N_{i} \boldsymbol{x}_{i}+\sum_{i=1}^{4} H_{i} \boldsymbol{x}_{i, \xi}
$$

Figure 2 Fifth-order Hermite contact finite element and its map to the current configuration of body $\mathcal{B}$

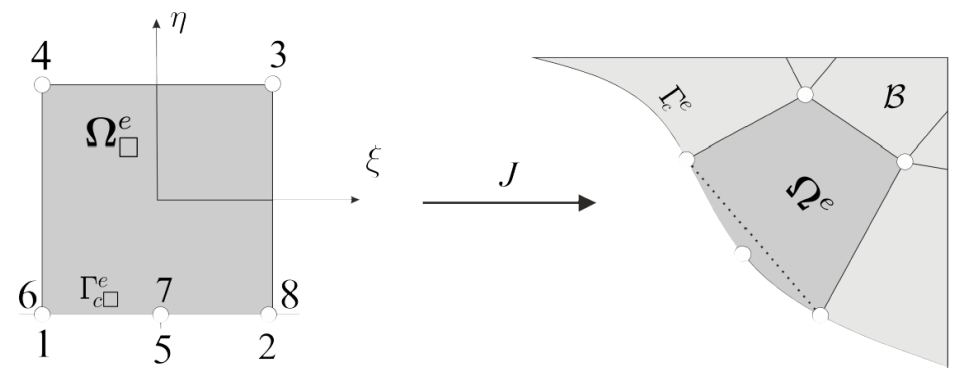

Figure 3 Representation of seventh-order Hermite enriched Q1CH7 contact finite element and its map to current configuration

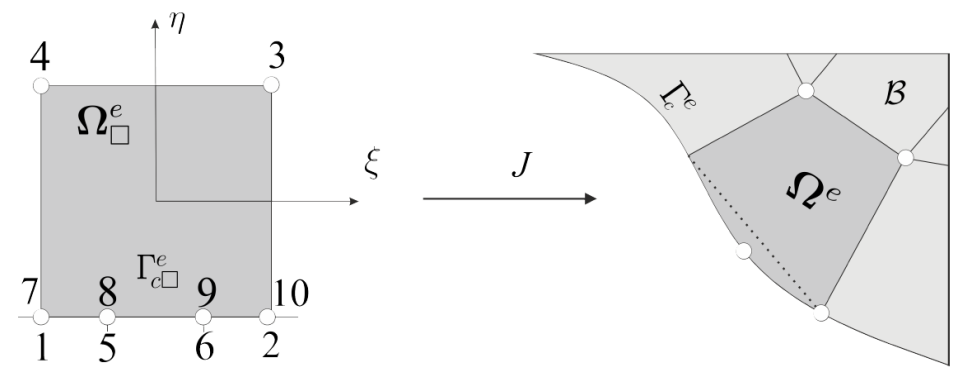

\subsection{Lagrangian enriched contact finite elements}

Lagrangian enrichment strategy presented in Sauer (2011) incorporates the higher-order Lagrangian basis functions for the enrichment of contact finite elements. In this paper, quadratic enriched (Q1C2) and quartic enriched (Q1C4) contact finite elements, introduced in Sauer (2011) are used to compare the obtained results with higher-order Hermite enriched contact finite elements.

\section{Numerical example}

To demonstrate the performance of proposed enriched contact finite elements a peeling of a deformable strip from a flat, rigid substrate, which is taken from Sauer (2011), is considered. Peeling is sometimes considered as tearing. However, in tearing two or more pieces are glued to each other and are pulled apart by external forces that might cause the damage or fracture in pieces during the motion. In this work, the strip and rigid substrate 
are held together by van der Waals adhesive forces. The strip initially lies at equilibrium distance to the substrate so that no adhesive forces act and equilibrium is maintained. When the external rotation angle $\theta$ is applied at its right end, it peels off the strip from the substrate without accounting any damage or fracture. The set-up of the problem is shown in Figure 4 and its detailed description is provided in the following.

Figure 4 The schematic arrangement of strip peeling problem

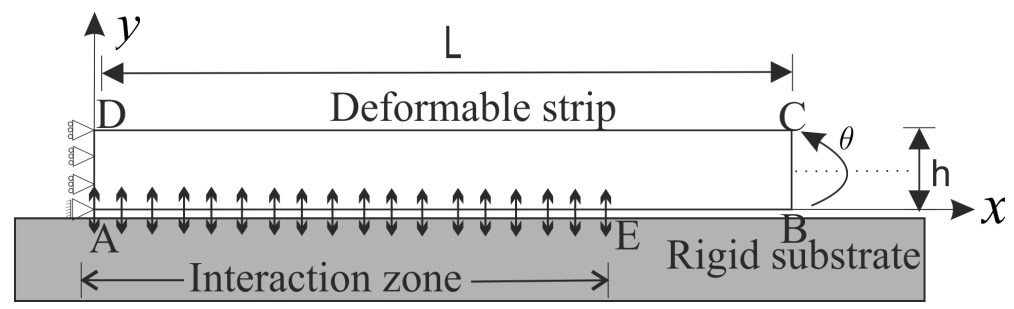

\subsection{Modelling parameters}

The dimension of the strip is $L \times h$, where length $L=200 L_{0}$, and height $h=10 L_{0}$ with $L_{0}=1 \mathrm{~nm}$. It is considered that adhesion is present at the $75 \%$ of the bottom surface of strip (from $x=0$ to $x=150 L_{0}$ ) and is calculated using equation (6) with $r_{0}=0.4 \mathrm{~nm}$ and $A_{H}=10^{-19} \mathrm{~J}$. For the modelling of strip, an isotropic nonlinear Neo-Hookean material model with $E=1 \mathrm{GPa}$ and $v=0.2$ under the plain strain conditions is used. The strips are discretised with the $240 \times 12,320 \times 16$ and $400 \times 20$ number of elements along the $\mathrm{x}$ - and $\mathrm{y}$-directions respectively and are referred as mesh 12, mesh 16, and mesh 20 arrangements in the further discussion.

\subsection{Application of peeling problem}

Peeling is considered to be central in many areas, e.g., coating, bonding, and adhesion. In our scope of work, the peeling of deformable strip having dimensions $L \times h$ and the value of adhesion parameters $r_{0}$ and $A_{H}$ are chosen such that they lead to the numerical simulation of the peeling process of gecko spatula adhesion (Sauer, 2011; Gautam and Sauer, 2013).

\subsection{Associated challenges}

In this work, the strip is modelled with the hyperelastic nonlinear material and undergoes the large deformation, which leads to the material and geometrical nonlinearities. Presence of these nonlinearities affects the quadratic-rate of convergence when solved with the Newton-Raphson method. Apart from them, the interaction forces, see equation (6), which are of highly nonlinear in nature as shows very high gradient even for a small change in distance between the interacting points, strongly affects the convergence-rate of Newton-Raphson iterations and leads to the non-physical or poor computational behaviour of peeling simulation, as discussed in Section 1. 


\subsection{Description of peeling process}

The strip is initially lying on the substrate in the equilibrium position and is peeled away from the substrate by externally applying the rotation angle $\theta$ to the right of its end, as shown in Figure 4. Its deformed configuration at different rotation angle is shown in Figure 9. For the bending of strip to $150^{\circ}$ from its initial configuration, a rotation step size $\Delta \theta=0.02$ is set for the simulation. The rotation angle $\theta$ is applied in such a manner that it yields a constant bending moment during the peeling of the strip from the aforementioned adhesion zone and is the theoretical solution (Sauer, 2011). With the application of rotation angle $\theta$, the strip is bent under the influence of van der Waals interaction forces. The finite element based system of equations is then solved with the Newton-Raphson iteration scheme for each rotation step $\Delta \theta$ and a resultant reaction force is determined. Using the values from the reaction force vector and displacement field for the right boundary's nodes of the strip, a corresponding reaction moment, which is considered as the bending moment for the strip, is computed.

Figure 5 A representative image of discretised domain of body $\mathcal{B}$ (using $n_{x}=40$ and $n_{y}=4$ ) along with the arrangement of contact elements (see online version for colours)

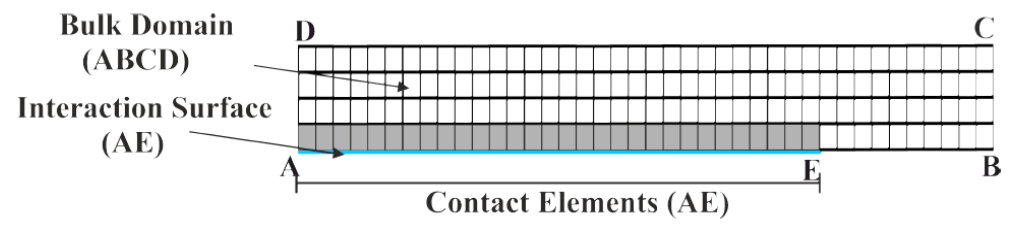

\subsection{Simulation with standard FE formulation}

In this case, the domain is discretised with the standard finite elements (i.e., denoted by Q1C1 notation) and during the numerical simulation it is observed that the computed bending moment increases linearly with the applied rotation $\theta$ for the peeling of first $25 \%$ of the length of strip (from $x=150 L_{0}$ to $x=200 L_{0}$ ) since no interaction is considered in this zone, see Figure 6(a). After a particular rotation angle onwards (approx. $60^{\circ}$ ), when the strip begins to peel off from the interaction zone of substrate, the bending moment at the right boundary of strip oscillate around a mean line rather than being constant (i.e., theoretical solution) as shown in Figure 6(b). The wavelength of the oscillation of bending moment is governed by the element length and oscillations are due to the inaccurate capturing of large gradient adhesive forces. The large amplitude and steep negative slopes of bending moment curve lead to quadratic convergence loss of the Newton-Raphson iterations. Refining the mesh lead to the reduction in the wavelength and amplitude of oscillations but comes at the expense of high computational cost. In this work, newly formulated contact enriched elements, Q1CH5 and Q1CH7, are used to address this issue, and the obtained results are provided in the following section. 
Figure 6 Comparison of bending moment oscillation for different enriched contact finite elements, (a) bending moment over the rotation angle $\theta$ for overall peeling process (b) zoomed view of bending moment curve shown in (a) (c) zoomed view of bending moment curve shown in (b) (see online version for colours)

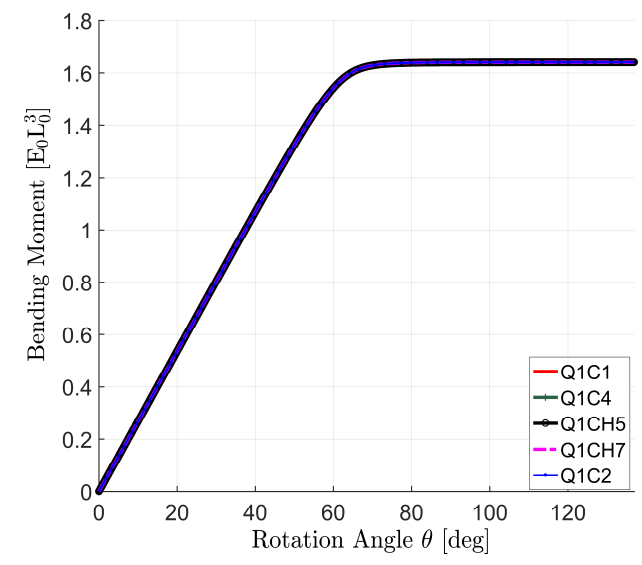

(a)

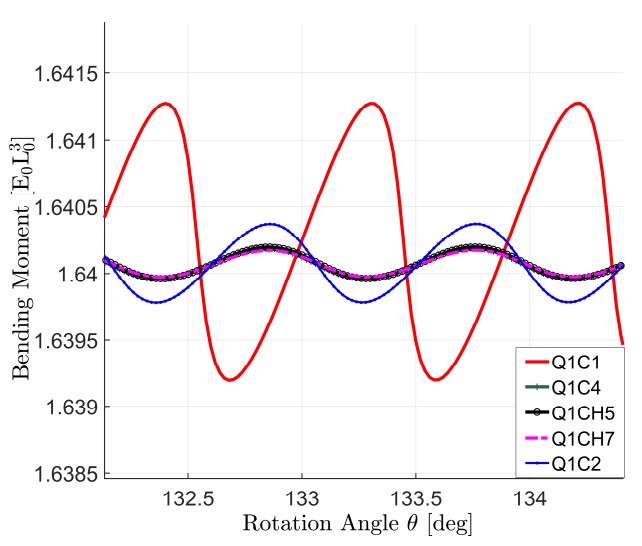

(b)

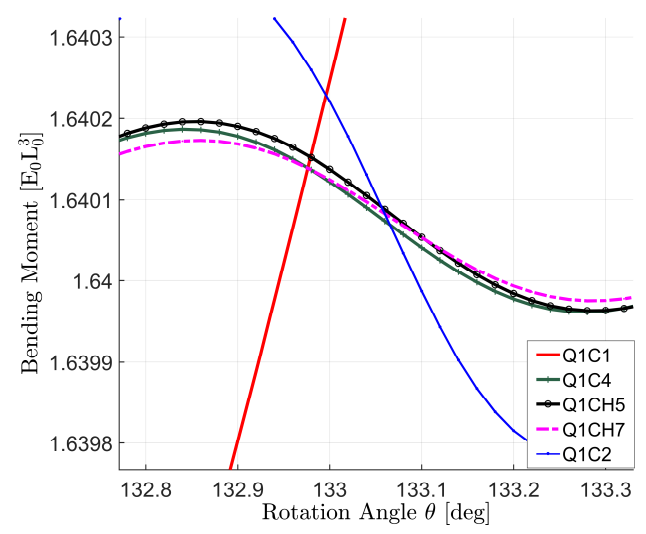

(c)

\section{Results and discussion}

The plot of bending moment over the applied rotation angle $\theta$ for the proposed enriched contact elements: Q1CH5 and Q1CH7 are shown in Figure 6. For the purpose of comparison, the bending moment curve performance of quadratic (Q1C2), and quartic-order (Q1C4) Lagrangian enriched contact finite element, presented by Sauer (2011), are also included. The illustrated results are first provided for the mesh 12 arrangement.

\subsection{Bending moment performance}

It can be observed that the newly formulated Q1CH7 element delivers the best result as it exhibits the lease oscillation error among the Q1C1, Q1C2, Q1C4 and Q1CH5 elements 
as shown in Figure 6(c). It reduces the amplitude of oscillation by a factor of 10.54, 2.96 and 1.15 and the negative slope to $11.14,4.36$ and 1.85 in comparison to Q1C1, Q1C2, and Q1C4 enrichment, respectively. The reduction in the oscillation error and downward slope leads to the robust performance of Newton-Raphson iterations and improves the accuracy of the computations. The amplitude of the moment curve oscillation and negative slope are further reduced when the domain is discretised with the mesh 16 and mesh 20 arrangements and is shown in Figures 7(a) and 7(b), respectively. The change in the amplitude of resulting bending moment curve over the rotation $\theta$ for different enrichment and discretisation is shown in Figure 8. As can be noticed that Q1CH7 element serves the best result and proposed enrichment yields the accurate, efficient, and improved results at the same mesh level in comparison to other enrichments. All the results have been obtained by running the code on the same machine. The deformed configuration of the strip at different rotation angle is shown in Figure 9.

Figure 7 Comparing the variation of bending moment curve for mesh 12, mesh 16, and mesh 20 arrangements (see online version for colours)

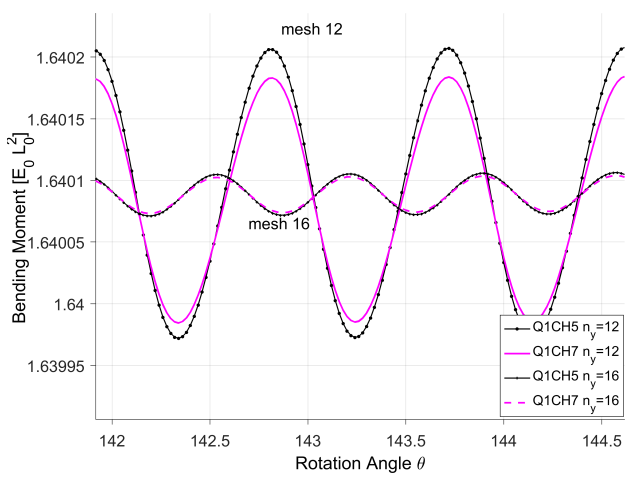

(a)

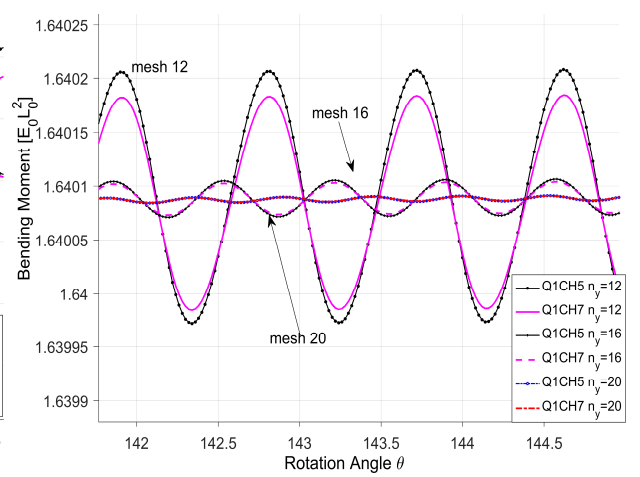

(b)

Figure 8 (a) Change in the amplitude of bending moment oscillation for different type of elements and discretisation (b) Zoomed view of amplitude curves shown in (a) (see online version for colours)

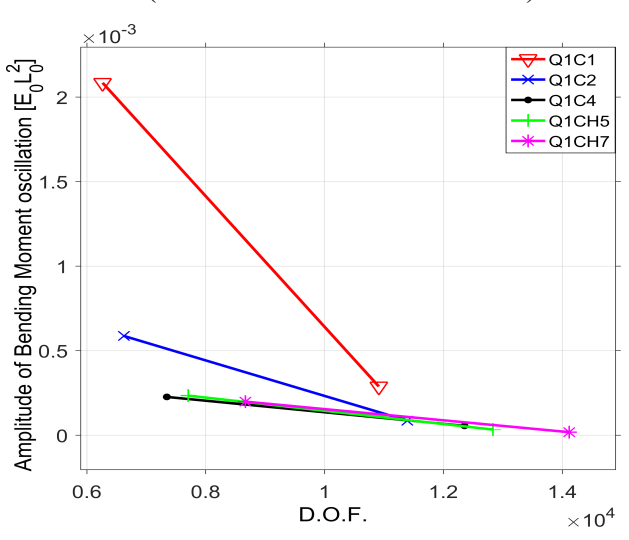

(a)

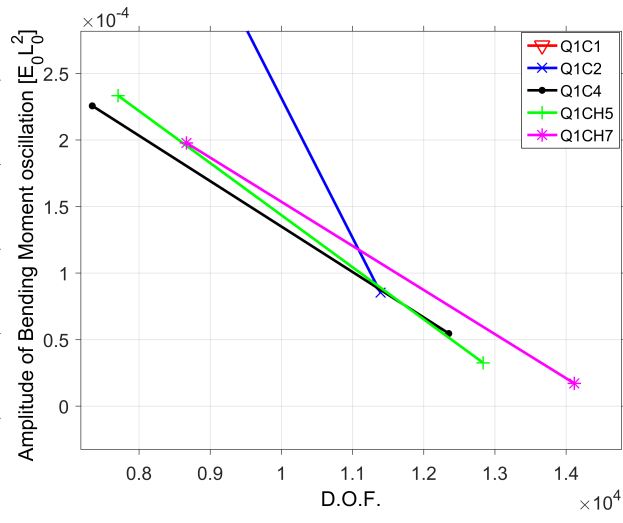

(b) 
Figure 9 Deformed configuration of the strip at various rotation angle during peeling, (a) $\theta=0^{\circ}$ (b) $\theta=30^{\circ}$ (c) $\theta=60^{\circ}$ (d) $\theta=90^{\circ}$ (e) $\theta=120^{\circ}$ (f) $\theta=150^{\circ}$ (see online version for colours)

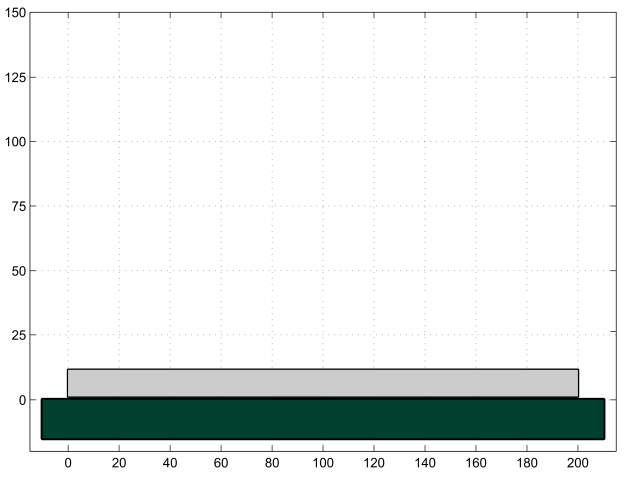

(a)

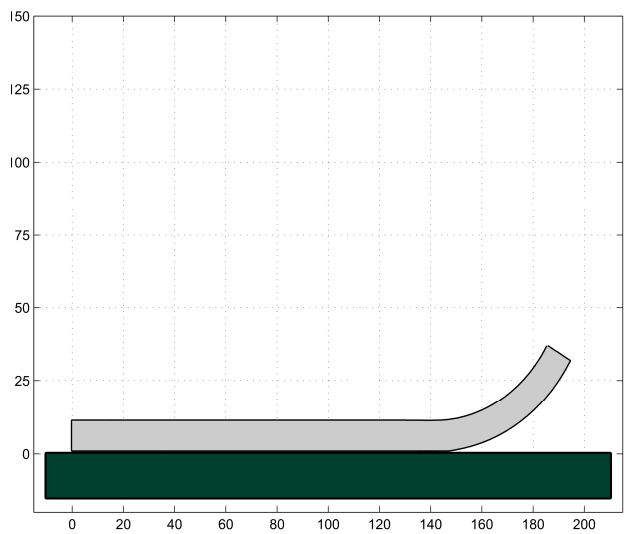

(c)

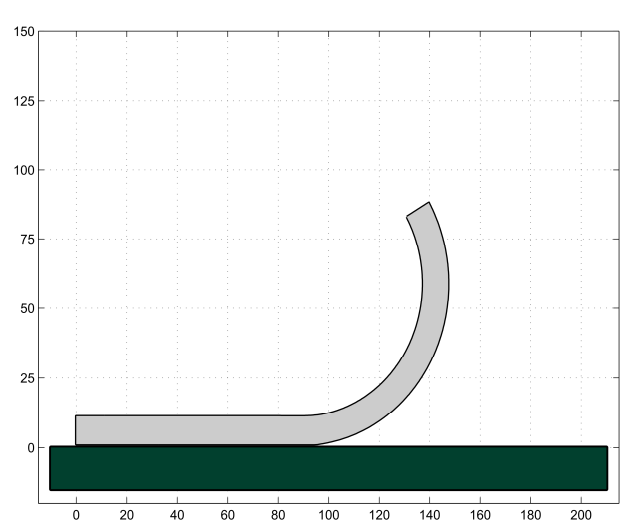

(e)

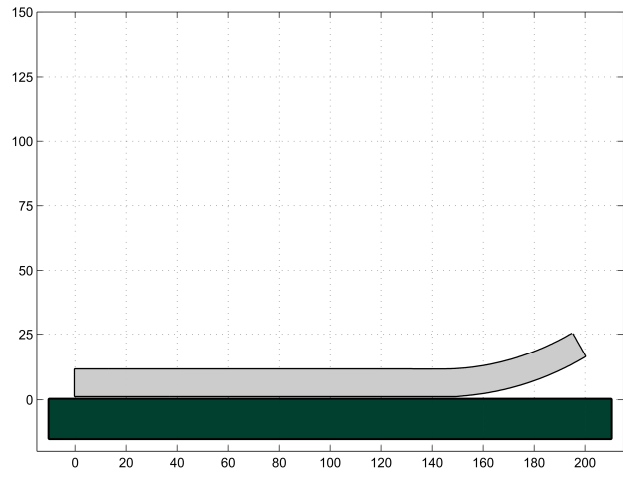

(b)

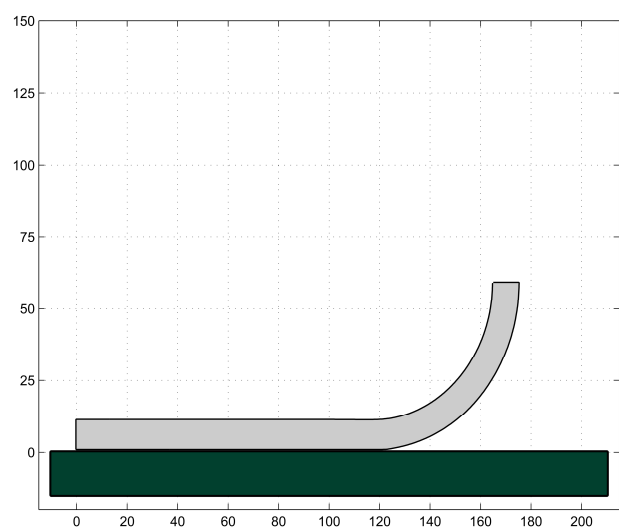

(d)

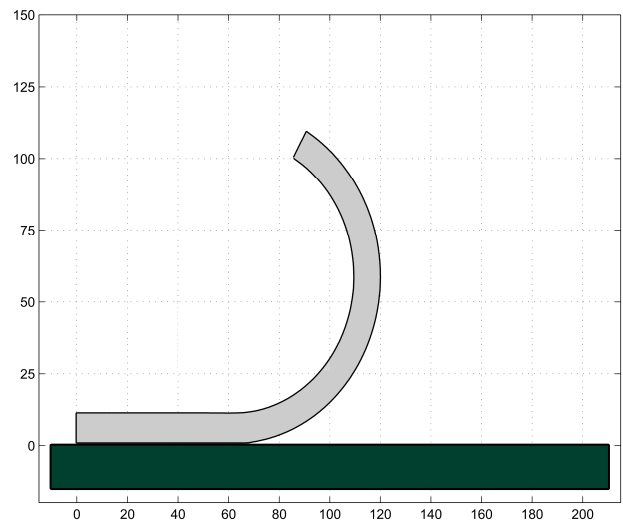

(f) 


\section{Conclusions}

During the numerical investigation of the adhesive contact problems, a highly oscillatory response of adhesive forces arises in the case of Lagrangian discretised FE formulation and causes the numerical difficulties. In this work, two new enriched contact finite elements are formulated to obtain the stable and improved result with respect to standard and higher-order Lagrangian enriched contact FE elements. The proposed elements, based on the inclusion of fifth- and seventh-order Hermite polynomial functions, exhibit the lowest oscillations error among all enriched contact finite elements. The reduction in the amplitude of peeling moment oscillation and negative slope improves the accuracy of adhesive contact computations at a coarser mesh resolution when compared with Lagrangian discretised FE formulation. For future work, the formulation and implementation of presented elements to three-dimensional finite elements are planned. Enrichment of contact elements with the NURBS basis functions within the framework of isogeometric analysis is also currently in progress.

\section{Acknowledgements}

The authors are grateful to the SERB, DST for supporting this research under project SR/FTP/ETA-0008/2014.

\section{References}

Augardea, C.E. (1998) 'Generation of shape functions for straight beam elements', Computers and Structures, Vol. 68, No. 6, pp.555-560.

Autumn, K., Sitti, M., Liang, Y.A., Peattie, A.M., Hansen, W.R., Sponberg, S., Kenny, T., Fearing, R., Israelachvili, J.N. and Full, R.J. (2002) 'Evidence for van der Waals adhesion in gecko setae', Proceedings National Academy of Sciences, Vol. 99, No. 19, pp.12252-12256.

Bonet, J. and Wood, R.D. (1997) Nonlinear Continuum Mechanics for Finite Element Analysis, Cambridge University Press, New York, USA.

Corbett, C.J. and Sauer, R.A. (2014) 'NURBS-enriched contact finite elements', Computer Methods in Applied Mechanics and Engineering, Vol. 275, pp.55-75.

Corbett, C.J. and Sauer, R.A. (2015) 'Three dimensional isogeometrically enriched finite elements for frictional contact and mixed-mode debonding', Computer Methods in Applied Mechanics and Engineering, Vol. 284, pp.781-806.

Gautam, S.S. and Sauer, R.A. (2013) 'An energy-momentum-conserving temporal discretization scheme for adhesive contact problems', International Journal for Numerical Methods in Engineering, Vol. 93, No. 10, pp.1057-1081.

Gautam, S.S. and Sauer, R.A. (2014) 'A composite time integration scheme for dynamic adhesion and its application to gecko spatula peeling', International Journal of Computational Methods, Vol. 11, No. 5, pp.1350104-1-1350104-28.

Ge, D. and Lu, L., Huang, X., Zhang, J., Qian, D., Zhang, L. and Li, Z. (2016) 'Elimination of boundary effect in silicon electrochemical etching via mechanical stress', International Journal of Materials and Structural Integrity, Vol. 10, No. 4, pp.170-180.

Hughes, T.J.R. (2000) The Finite Element Method: Linear Static and Dynamic Finite Element Analysis, Dover Publications, Mineola, New York.

Israelachvili, J.N. (1991) Intermolecular and Surface Forces, Academic Press, London. 
Laursen, T.A. (2002) Computational Contact and Impact Mechanics: Fundamentals of Modeling Interfacial Phenomena in Nonlinear Finite Element Analysis, Springer, Berlin.

Sauer, R.A. (2008) 'An atomic interaction-based rod formulation for modelling Gecko adhesion', PAMM, WILEY-VCH Verlag, Vol. 8, No, 1, pp.10193-10194.

Sauer, R.A. (2011) 'Enriched contact finite elements for stable peeling computations', International Journal for Numerical Methods in Engineering, Vol. 87, No. 6, pp.593-616.

Sauer, R.A. and Holl, M. (2013) 'A detailed 3D finite element analysis of the peeling behaviour of a gecko spatula', Computer Methods in Biomechanics and Biomedical Engineering, Vol. 16, No. 6, pp.577-591.

Sauer, R.A. and Li, S. (2007a) 'An atomic interaction-based continuum model for adhesive contact mechanics', Finite Elements in Analysis and Design, Vol. 43, No. 5, pp.384-396.

Sauer, R.A. and Li, S. (2007b) 'A contact mechanics model for quasi-continua', International Journal for Numerical Methods in Engineering, Vol. 71, No. 8, pp.931-962.

Sauer, R.A. and Lorenzis, L.D. (2013) 'A computational contact formulation based on surface potentials', Computer Methods in Applied Mechanics and Engineering, Vol. 253, pp.369-395.

Sauer, R.A. and Wriggers, P. (2009) 'Formulation and analysis of a 3D finite element implementation for adhesive contact at nanoscale', Computer Methods in Applied Mechanics and Engineering, Vol. 198, Nos. 49-52, pp.3871-3883.

Wriggers, P. (2006) Computational Contact Mechanics, Springer, Berlin. 